\title{
MULTIPLICATIVE FUNCTIONS ON ARITHMETIC PROGRESSIONS
}

\author{
ADOLF HILDEBRAND \\ (Communicated by William Adams)
}

\begin{abstract}
Let $f$ be a multiplicative arithmetic function satisfying $|f| \leq 1$, let $x \geq 10$ and $2 \leq Q \leq x^{1 / 3}$. It is shown that, with suitable integers $q_{1} \geq 2$
\end{abstract} and $q_{2} \geq 2$, the estimate

$$
\sum_{\substack{n \leq x \\ n \equiv a \bmod q}} f(n)=\frac{1}{\varphi(q)} \sum_{\substack{n \leq x \\(n, q)=1}} f(n)+O\left(\frac{x}{q}\left(\log \frac{\log x}{\log Q}\right)^{-1 / 2}\right)
$$

holds uniformly for $(a, q)=1$ and all moduli $q \leq Q$ that are not multiples of $q_{1}$ or $q_{2}$.

\section{INTRODUCTION}

To investigate the distribution of an arithmetic function $f: \mathbf{N} \rightarrow \mathbf{C}$ among arithmetic progressions, it is natural to consider the quantities

$$
\Delta(x ; q, a)=\Delta_{f}(x ; q, a)=\sum_{\substack{n \leq x \\ n \equiv a \bmod q}} f(n)-\frac{1}{\varphi(q)} \sum_{\substack{n \leq x \\(n, q)=1}} f(n)
$$

and

$$
\Delta(x ; q)=\Delta_{f}(x ; q)=\max _{(a, q)=1}|\Delta(x ; q, a)| .
$$

The goal is to obtain upper bounds that are valid uniformly with respect to $q$ in as large a range as possible.

In the case when $f$ is the characteristic function of all primes, this reduces to a classical problem in prime number theory, and several fundamental results are known. The Siegel-Walfisz Theorem gives in this case a non-trivial upper bound for moduli $q$ that are less than arbitrary, but fixed, power of $\log x$. The Bombieri-Vinogradov Theorem shows that such a bound remains valid on average in the much larger range $q \leq x^{1 / 2-\varepsilon}$. In addition to these well-known results there is a result of Gallagher [7] which yields a non-trivial estimate for all moduli $q$ up to a certain power of $x$, with the possible exception of the

Received by the editors September 3, 1988 and, in revised form, March 31, 1989.

1980 Mathematics Subject Classification (1985 Revision). Primary 11 N37.

Supported by National Science Foundation grant DMS 8640693. 
multiples of some "exceptional" modulus $q_{0}$. Specifically, Gallagher's theorem implies that if $f$ is the characteristic function of the primes then

$$
\Delta_{f}(x ; q) \ll \frac{x}{\varphi(q) \log x}\left\{\exp \left(-c_{1} \frac{\log x}{\log Q}\right)+\exp \left(-c_{1} \sqrt{\log x}\right)\right\}
$$

holds with some positive constant $c_{1}$ uniformly for $2 \leq Q \leq \sqrt{x}$ and all $q \leq Q$, except possibly the multiples of a certain $q_{0}=q_{0}(x, Q) \geq 2$.

Analogues of these results, and in particular of the Bombieri-Vinogradov Theorem, have first been obtained for certain other special functions $f$ like the Moebius function or the divisor functions; see, for example, Wolke [12]. More recently, Bombieri, Friedlander and Iwaniec [2] (see also [1]), using an idea of Motohashi [9], gave a very general Bombieri-Vinogradov type theorem for functions $f$ that can be represented as convolutions of "well-behaved" functions. Elliott [3, Chapter 7] [4] and the author [8] showed that all additive functions satisfy a Bombieri-Vinogradov type theorem.

Very recently, Elliott [5] [6] considered the case of multiplicative functions $f$ satisfying $|f| \leq 1$. He showed that for such functions $f$ one has

$$
\Delta_{f}(x ; q) \ll\left(\frac{\log \log x}{\log x}\right)^{1 / 8} x
$$

for all $q$ except possibly the multiples of a certain $q_{1}=q_{1}(f, x) \geq 2$. The estimate (2) constitutes an improvement over the trivial bound $\Delta_{f}(x ; q) \ll x / q$ in the range $q \leq(\log x)^{1 / 8-\varepsilon}$, and thus can be regarded as a (partial) analogue of the Siegel-Walfisz Theorem. Elliott [6] also proved a Bombieri-Vinogradov type theorem for multiplicative functions of modulus $\leq 1$. The purpose of this paper is to complement these results by the following theorem that represents a (partial) analogue of the above-mentioned theorem of Gallagher.

Theorem. Let $f$ be a multiplicative function satisfying $|f| \leq 1$, let $x \geq 10$ and $2 \leq Q \leq x^{1 / 3}$. Then there exist integers $q_{1} \geq 2$ and $q_{2} \geq 2$ such that the bound

$$
\Delta_{f}(x ; q) \ll \frac{x}{q}\left(\log \frac{\log x}{\log Q}\right)^{-1 / 2}
$$

holds for all moduli $q \leq Q$ that are not multiples of $q_{1}$ or $q_{2}$.

The logarithmic factor in (3) represents the saving over the trivial bound $\ll x / q$. Compared to Gallagher's estimate (1), the saving is relatively modest, but this is not surprising in view of the rather general hypotheses in the theorem. Note that (3) is non-trivial in essentially the same range as (1), namely for all values $Q$ that do not exceed a certain fixed power of $x$.

Of the two "exceptional" moduli $q_{1}$ and $q_{2}$ in the theorem, one plays the same role as in Elliott's result; it is necessary, for example, in the case when $f$ is equal to a nonprincipal Dirichlet character. The other exceptional modulus is connected with possible Siegel zeros and closely related to the exceptional 
modulus in Gallagher's theorem. If there are no Siegel zeros, then this modulus can be dropped.

To prove the theorem we shall use an essentially elementary approach based on the large sieve that is different from the methods of both Elliott and Gallagher.

\section{Preliminaries}

We gather here some (variants of) known results that we shall use in the proof of the theorem. The first lemma is a special case of the large sieve in its arithmetic form and can be found in Elliott [3, p. 105].

Lemma 1. Let $N$ be a positive integer and $a_{n}(n=1, \ldots, N)$ be arbitrary complex numbers. Then we have

$$
\sum_{p \leq \sqrt{N}} \frac{1}{p} \sum_{r=1}^{p}\left|p \sum_{\substack{1 \leq n \leq N \\ n \equiv r \bmod p}} a_{n}-\sum_{1 \leq n \leq N} a_{n}\right|^{2} \ll N \sum_{1 \leq n \leq N}\left|a_{n}\right|^{2},
$$

where the implied constant is absolute.

The next result is a simple consequence of the theorem of Gallagher [7] referred to in the introduction; for the argument see, for example, Wirsing [11, Lemma 1].

Lemma 2. There exists a constant $\gamma>0$ such that the estimate

$$
\pi(x+h ; q, a)-\pi(x ; q, a) \asymp \frac{h}{\varphi(q) \log x}
$$

holds uniformly for all sufficiently large $x, x \exp (-\sqrt{\log x}) \leq h \leq x, q \leq x^{\gamma}$ and $(a, q)=1$, with the possible exception of those moduli $q$ that are divisible by a certain $q_{0}=q_{0}(x) \geq 2$.

Finally, we shall need the following Brun-Titchmarsh type theorem for multiplicative functions which is essentially due to Shiu [10].

Lemma 3. Let $\varepsilon>0$ be fixed, and let $f$ be a multiplicative function satisfying $0 \leq f \leq 1$. Then we have, for all $x \geq 3, q \leq x^{1-\varepsilon}$ and $(a, q)=1$,

$$
\sum_{\substack{n \leq x \\ n \equiv a \bmod q}} f(n) \ll_{\varepsilon} \frac{x}{q} \exp \left\{-\sum_{\substack{p \leq x \\ p \nmid q}} \frac{1-f(p)}{p}\right\},
$$

where the implied constant depends at most on $\varepsilon$.

Shiu actually showed that under more general assumptions on $f$ one has

$$
\sum_{\substack{n \leq x \\ n \equiv a \bmod q}} f(n) \ll_{\varepsilon} \frac{x}{\varphi(q) \log x} \exp \left\{\sum_{\substack{p \leq x \\ p \nmid q}} \frac{f(p)}{p}\right\} .
$$


The lemma follows from this, since

$$
\frac{1}{\log x} \ll \exp \left\{-\sum_{p \leq x} \frac{1}{p}\right\}
$$

and

$$
\frac{1}{\varphi(q)}=\frac{1}{q} \prod_{p \mid q}\left(1-\frac{1}{p}\right)^{-1} \ll \frac{1}{q} \exp \left\{\sum_{p \mid q} \frac{1}{p}\right\}
$$

\section{Proof of THE THeOReM}

We fix a multiplicative function $f$ and real numbers $x$ and $Q$ as in the theorem. Without loss of generality we may assume that $x$ is sufficiently large and that $Q$ lies in the range

$$
\exp (\sqrt{\log x}) \leq Q \leq x^{\gamma / 10}
$$

where $\gamma$ is the constant in Lemma 2.

We normalize the quantities $\Delta(x ; q, a)$ and $\Delta(x ; q)$ by setting

$$
\delta(x ; q, a)=\frac{q}{x} \Delta(x ; q, a)=\frac{q}{x} \sum_{\substack{n \leq x \\ n \equiv a \bmod q}} f(n)-\frac{q}{\varphi(q) x} \sum_{\substack{n \leq x \\(n, q)=1}} f(n)
$$

and

$$
\delta(x ; q)=\max _{(a, q)=1}|\delta(x ; q, a)| .
$$

More generally, if $q^{\prime}$ is a multiple of $q$, then we define

$$
\delta^{\left(q^{\prime}\right)}(x ; q, a)=\frac{\varphi(q)}{\varphi\left(q^{\prime}\right)} \sum_{\substack{1 \leq a^{\prime} \leq q^{\prime} \\\left(a^{\prime}, q^{\prime}\right)=1 \\ a^{\prime} \equiv a \bmod q}} \delta\left(x ; q^{\prime}, a^{\prime}\right)
$$

and

$$
\delta^{\left(q^{\prime}\right)}(x ; q)=\max _{(a, q)=1}\left|\delta^{\left(q^{\prime}\right)}(x ; q, a)\right| .
$$

It is easy to see that the sum in (7) has exactly $\varphi\left(q^{\prime}\right) / \varphi(q)$ terms if $(a, q)=1$ and $q \mid q^{\prime}$. Thus (7) defines $\delta^{\left(q^{\prime}\right)}(x ; q, a)$ as an average over a subset of the numbers $\delta\left(x ; q^{\prime}, a^{\prime}\right),\left(a^{\prime}, q^{\prime}\right)=1$, and it follows in particular that

$$
\delta^{\left(q^{\prime}\right)}(x ; q) \leq \delta\left(x ; q^{\prime}\right) .
$$

We next derive a simple upper bound for the quantities $\delta$ and $\delta^{\left(q^{\prime}\right)}$. Suppose that $x^{1 / 2} \leq y \leq x$ and $q \leq Q$, so that $q \leq x^{\gamma / 10} \leq x^{1 / 10} \leq y^{1 / 5}$ by (5). Using 
(6), Cauchy's inequality and Lemma 3 , we find

$$
\begin{aligned}
\delta(y ; q)^{2} & \ll \max _{(a, q)=1}\left|\frac{q}{y} \sum_{\substack{n \leq y \\
n \equiv a \bmod q}} f(n)\right|^{2} \ll \max _{(a, q)=1} \frac{q}{y} \sum_{\substack{n \leq y \\
n \equiv a \bmod q}}|f(n)|^{2} \\
& \ll \exp \left\{-\sum_{\substack{p \leq y \\
p \nmid q}} \frac{1-|f(p)|^{2}}{p}\right\} \ll e^{-S},
\end{aligned}
$$

where

$$
S=\sum_{Q<p \leq x} \frac{1-|f(p)|^{2}}{p} .
$$

Thus, by (8), it follows that

$$
\delta^{\left(q^{\prime}\right)}(y ; q) \ll e^{-S / 2}
$$

holds uniformly for $x^{1 / 2} \leq y \leq x, q^{\prime} \leq Q$ and $q \mid q^{\prime}$.

The following lemma gives a functional relation between the quantities $\delta^{\left(q^{\prime}\right)}(y ; q, r)$ that will form the basis of our argument.

Lemma 4. Let $q^{\prime} \leq Q, q \mid q^{\prime}$ and $(a, q)=1$. Then we have

$$
\sum_{Q<p \leq x^{1 / 4}} \frac{1}{p}\left|\delta^{\left(q^{\prime}\right)}(x ; q, a)-f(p) \delta^{\left(q^{\prime}\right)}\left(\frac{x}{p} ; q, a \bar{p}\right)\right|^{2} \ll 1,
$$

where $\bar{p}$ is the inverse of $p$ modulo $q$.

Proof. Since $\delta^{\left(q^{\prime}\right)}(x / p ; q, a \bar{p})$ depends only on the residue class of $a \bar{p}$ modulo $q$ and $q \mid q^{\prime}$, we may assume that $\bar{p}$ is an inverse of $p$ modulo $q^{\prime}$. In view of the inequality

$$
\begin{aligned}
& \left|\delta^{\left(q^{\prime}\right)}(x ; q, a)-f(p) \delta^{\left(q^{\prime}\right)}\left(\frac{x}{p} ; q, a \bar{p}\right)\right|^{2} \\
& \quad=\left|\frac{\varphi(q)}{\varphi\left(q^{\prime}\right)} \sum_{\substack{\left(a^{\prime}, q^{\prime}\right)=1 \\
a^{\prime} \equiv a \bmod q}}\left(\delta\left(x ; q^{\prime}, a^{\prime}\right)-f(p) \delta\left(\frac{x}{p} ; q^{\prime}, a^{\prime} \bar{p}\right)\right)\right|^{2} \\
& \quad \leq \frac{\varphi(q)}{\varphi\left(q^{\prime}\right)} \sum_{\substack{\left(a^{\prime}, q^{\prime}\right)=1 \\
a^{\prime} \equiv a \bmod q}}\left|\delta\left(x ; q^{\prime}, a^{\prime}\right)-f(p) \delta\left(\frac{x}{p} ; q^{\prime}, a^{\prime} \bar{p}\right)\right|^{2},
\end{aligned}
$$

which follows from (7), it suffices to prove (11) in the case $q=q^{\prime}$, i.e., with $\delta(x ; q, a)$ in place of $\delta^{\left(q^{\prime}\right)}(x ; q, a)$. We may also suppose that $a$ satisfies $1 \leq a \leq q$. 
We shall apply Lemma 1 with $N=[x / q]$ and $a_{n}=f(n q+a)$. Note that $N \geq[x / Q] \geq\left[x^{1-\gamma / 10}\right] \geq x^{9 / 10}$ by (5), so that $N^{1 / 2} \geq x^{1 / 4}$ if $x$ is sufficiently large, as we may assume. Thus the range of summation in (11) is contained in that of the outer sum in (4). By our assumptions $|f| \leq 1$ and $1 \leq a \leq q$ and the hypothesis $q \leq Q \leq x^{\gamma / 10}$ the right-hand side of (4) is

$$
N \sum_{n \leq N}\left|a_{n}\right|^{2} \leq \frac{x}{q} \sum_{n \leq x / q}|f(n q+a)|^{2} \ll\left(\frac{x}{q}\right)^{2},
$$

and we have

$$
\sum_{n \leq N} a_{n}=\sum_{n \leq x / q} f(n q+a)=\sum_{\substack{n \leq x \\ n \equiv a \bmod q}} f(n)+O(1) .
$$

Moreover, if $p$ is a prime in the range $Q<p \leq x^{1 / 4}$ and $1 \leq r \leq p$, then

$$
\sum_{\substack{n \leq N \\ n \equiv r \bmod p}} a_{n}=\sum_{\substack{n \leq x \\ n \equiv a \bmod q \\ n=q r+a \bmod p}} f(n)+O(1) .
$$

Choosing $r=r_{p}$ such that $q r+a \equiv 0 \bmod p$ (which is possible, since $(q, p)=1$ for $p>Q \geq q$ ), the last sum becomes

$$
\sum_{\substack{n p \leq x \\ n p \equiv a \bmod q}} f(n p)=f(p) \sum_{\substack{n \leq x / p \\ n \equiv a \overline{\bar{p}} \bmod q}} f(n)+O\left(\frac{x}{p^{2} q}\right)+O(1)
$$

by the multiplicativity of $f$. Thus, taking in (4) only the terms with $Q<p \leq$ $x^{1 / 4}$ and $r=r_{p}$, we obtain

$$
\sum_{Q<p \leq x^{1 / 4}} \frac{|E(p, a)|^{2}}{p} \ll\left(\frac{x}{q}\right)^{2}+\sum_{Q<p \leq x^{1 / 4}} \frac{1}{p}\left(1+\frac{x}{p^{2} q}\right)^{2} \ll\left(\frac{x}{q}\right)^{2},
$$

where

$$
E(p, a)=\sum_{\substack{n \leq x \\ n \equiv a \bmod q}} f(n)-p f(p) \sum_{\substack{n \leq x / p \\ n \equiv a \bar{p} \bmod q}} f(n) .
$$

Now, by (6) we have

$$
\delta(x ; q, a)-f(p) \delta\left(\frac{x}{p} ; q, a \bar{p}\right)=\left(\frac{q}{x}\right)\left(E(p, a)-\frac{1}{\varphi(q)} \sum_{(b, q)=1} E(p, b)\right),
$$

whence

$$
\left|\delta(x ; q, a)-f(p) \delta\left(\frac{x}{p} ; q, a \bar{p}\right)\right| \ll\left(\frac{q}{x}\right)^{2}\left\{|E(p, a)|^{2}+\frac{1}{\varphi(q)} \sum_{(b, q)=1}|E(p, b)|^{2}\right\} .
$$


We therefore get for the left-hand side of (11) (with $q^{\prime}=q$ ) the bound

$$
\ll\left(\frac{q}{x}\right)^{2}\left\{\sum_{Q<p \leq x^{1 / 4}} \frac{|E(p, a)|^{2}}{p}+\frac{1}{\varphi(q)} \sum_{(b, q)=1} \sum_{Q<p \leq x^{1 / 4}} \frac{|E(p, b)|^{2}}{p}\right\},
$$

which is $\ll 1$ by (12). This proves the lemma.

Let $q_{0}=q_{0}(x)$ denote the "exceptional" modulus of Lemma 2 and set $Q_{1}=$ $Q^{2 / \gamma}$, so that $Q_{1} \leq x^{1 / 5}$ by (5).

Lemma 5. Let $q, q_{1}^{\prime} \leq Q, q\left|q^{\prime}, q_{1}\right| q_{1}^{\prime}$ and set $d=\left(q, q_{1}\right), D=\left[q, q_{1}\right]$. If $q_{0} \nmid D$, then we have, for $(a, q)=1,\left(a_{1}, q_{1}\right)=1$, and any complex number $\lambda$,

$$
\begin{aligned}
& \sum_{Q_{1}<p \leq x^{1 / 4}} \frac{1}{p}\left|\delta^{\left(q^{\prime}\right)}\left(\frac{x}{p} ; q, a \bar{p}\right)-\delta^{\left(q^{\prime}\right)}\left(\frac{x}{p} ; d, a \bar{p}\right)\right|^{2} \\
& \ll \sum_{Q_{1}<p \leq x^{1 / 4}} \frac{1}{p}\left|\delta^{\left(q^{\prime}\right)}\left(\frac{x}{p} ; q, a \bar{p}\right)-\lambda \delta^{\left(q^{\prime}\right)}\left(\frac{x}{p} ; q_{1}, a_{1} \bar{p}\right)\right|^{2}+1+|\lambda|^{2},
\end{aligned}
$$

where in $\delta^{\left(q^{\prime}\right)}(y ; k, b \bar{p}), \bar{p}$ is understood to be defined with respect to the modulus $k$.

Proof. Let $I$ denote an interval of the type

$$
I=\left(y, y\left(1+\frac{1}{\log x}\right)\right], \quad Q_{1}<y \leq x^{1 / 4} .
$$

We shall show that for every such interval we have

$$
\begin{aligned}
& \sum_{p \in I}\left|\delta^{\left(q^{\prime}\right)}\left(\frac{x}{p} ; q, a \bar{p}\right)-\delta^{\left(q^{\prime}\right)}\left(\frac{x}{p} ; d, a \bar{p}\right)\right|^{2} \\
& \ll \sum_{p \in I}\left|\delta^{\left(q^{\prime}\right)}\left(\frac{x}{p} ; q, a \bar{p}\right)-\lambda \delta^{\left(q^{\prime}\right)}\left(\frac{x}{p} ; q_{1}, a_{1} \bar{p}\right)\right|^{2}+O\left(\left(1+|\lambda|^{2}\right) \sum_{p \in I} \frac{1}{\log x}\right) .
\end{aligned}
$$

Since $1 / p \asymp 1 / y$ for $p \in I$, this relation remains valid if we insert a factor $1 / p$ into each of the three sums. We then obtain (13) by subdividing the interval $\left(Q_{1}, x^{1 / 4}\right.$ ] into intervals of type $\left(^{*}\right)$ (with a possible overlap of two such intervals) and noting that

$$
\sum_{Q_{1}<p \leq x^{1 / 4}} \frac{1}{p \log x} \ll 1
$$

To prove (14) we shall exploit the fact that the functions $\delta$ essentially depend only on the residue classes of $p$ modulo $q$ and modulo $q_{1}$, as long as $p$ is confined to an interval of the above type. Indeed, from (7) and (6) it readily follows that if $p \in I$ and $(r, q)=1$, then

$$
\delta^{\left(q^{\prime}\right)}\left(\frac{x}{p} ; q, a \bar{r}\right)=\delta^{\left(q^{\prime}\right)}\left(\frac{x}{y} ; q, a \bar{r}\right)+O\left(\frac{1}{\log x}\right)=a(r)+O\left(\frac{1}{\log x}\right),
$$


say, and similarly

$$
\begin{aligned}
\delta^{\left(q^{\prime}\right)}\left(\frac{x}{p} ; q_{1}, a \bar{r}\right) & =b(r)+O\left(\frac{1}{\log x}\right), \\
\delta^{\left(q^{\prime}\right)}\left(\frac{x}{p} ; d, a \bar{r}\right) & =c(r)+O\left(\frac{1}{\log x}\right),
\end{aligned}
$$

with

$$
\begin{aligned}
& b(r)=\delta^{\left(q^{\prime}\right)}\left(\frac{x}{y} ; q_{1}, a_{1} \bar{r}\right), \\
& c(r)=\delta^{\left(q^{\prime}\right)}\left(\frac{x}{y} ; d, a \bar{r}\right) .
\end{aligned}
$$

The functions $a(r), b(r)$, and $c(r)$ are periodic with periods $q, q_{1}$, and $d$ (as well as $q$ ), respectively. Replacing the functions $\delta$ in (14) by these approximations introduces an error term of order

$$
\ll\left(1+|\lambda|^{2}\right) \sum_{p \in I} \frac{1}{\log x}
$$

on each side, which is acceptable. Thus, to prove (14) it will be enough to show

$$
\sum_{p \in I}|a(p)-c(p)|^{2} \ll \sum_{p \in I}|a(p)-\lambda b(p)|^{2} .
$$

Because of the periodicity of the functions $a(p), b(p)$ and $c(p)$ the two sides of (15) can be written as

$$
\sum_{1 \leq r_{1} \leq q_{1}} \sum_{1 \leq r \leq q}|a(r)-c(r)|^{2} N\left(r, r_{1}\right)
$$

and

$$
\sum_{1 \leq r_{1} \leq q_{1}} \sum_{1 \leq r \leq q}\left|a(r)-\lambda b\left(r_{1}\right)\right|^{2} N\left(r, r_{1}\right),
$$

respectively, where

$$
N\left(r, r_{1}\right)=\#\left\{p \in I: p \equiv r \bmod q, p \equiv r_{1} \bmod q_{1}\right\} .
$$

Clearly, $N\left(r, r_{1}\right)=0$, unless $(r, q)=1,\left(r_{1}, q_{1}\right)=1$ and $r \equiv r_{1} \bmod d$, and if these conditions are satisfied, then we have

$$
\begin{aligned}
N\left(r, r_{1}\right) & =\#\{p \in I: p \equiv s \bmod D\} \\
& =\pi\left(y\left(1+\frac{1}{\log x}\right) ; D, s\right)-\pi(y ; D, s)
\end{aligned}
$$

with $D=\left[q, q_{1}\right]$ and a suitable $s,(s, D)=1$. We can estimate this last quantity by Lemma 2 , since $q_{0} \nmid D, D \leq q q_{1} \leq Q^{2}-Q_{1}^{\gamma} \leq y^{\gamma}$ by the hypotheses of Lemma 5, and

$$
y \geq \frac{y}{\log x} \geq \frac{x}{(\log Q)^{2}} \geq \frac{y}{(\log y)^{2}} \geq y \exp (-\sqrt{\log y})
$$


by (5), if we assume, as we may, that $x$ and $y$ are sufficiently large. We then obtain the bound

$$
N\left(r, r_{1}\right) \asymp \frac{y}{\varphi(D) \log y \log x}
$$

in the case $(r, q)=1,\left(r_{1}, q_{1}\right)=1$ and $r \equiv r_{1} \bmod d$. Thus $N\left(r, r_{1}\right)$ has the same order of magnitude for such pairs $\left(r, r_{1}\right)$, and is zero otherwise. To prove (15), it therefore suffices to show that

$$
\sum_{\left(r_{1}, q_{1}\right)=1} \sum_{\substack{(r, q)=1 \\ r \equiv r_{1} \bmod d}}|a(r)-c(r)|^{2} \ll \sum_{\left(r_{1}, q_{1}\right)=1} \sum_{\substack{(r, q)=1 \\ r \equiv r_{1} \bmod d}}\left|a(r)-\lambda b\left(r_{1}\right)\right|^{2} .
$$

Note that $c(r)$ depends only on the residue class of $r$ modulo $d$, so that $c(r)=c\left(r_{1}\right)$ in (16). More precisely, (7) shows that $c\left(r_{1}\right)$ is the arithmetic mean of the numbers $a(r)$, where $1 \leq r \leq q,(r, q)=1$ and $r \equiv r_{1} \bmod d$. It follows that for each $r_{1}$ with $\left(r_{1}, q_{1}\right)=1$ we have

$$
\begin{aligned}
\sum_{\substack{(r, q)=1 \\
r \equiv r_{1} \bmod d}}|a(r)-c(r)|^{2} & =\sum_{\substack{\left(r_{1}, q\right)=1 \\
r \equiv r_{1} \bmod d}}\left|a(r)-c\left(r_{1}\right)\right|^{2} \\
& =\min _{\mu} \sum_{\substack{(r, q)=1 \\
r \equiv r_{1} \bmod d}}|a(r)-\mu|^{2} \\
& \leq \sum_{\substack{(r, q)=1 \\
r \equiv r_{1} \bmod d}}\left|a(r)-\lambda b\left(r_{1}\right)\right|^{2} .
\end{aligned}
$$

Summing this inequality over $\left(r_{1}, q_{1}\right)=1$ yields (16) and thus completes the proof of Lemma 5.

Lemma 6. Let $q, q_{1}, q_{1}^{\prime}$ and $d=\left(q, q_{1}\right)$ be as in Lemma 5. Then we have

$$
\delta^{(q)}(x ; q) \leq c\left\{\delta^{(q)}(x ; d)+\frac{1}{\sqrt{\mathscr{L}}}+\frac{\delta^{\left(q^{\prime}\right)}(x ; q)}{\sqrt{\mathscr{L}} \delta^{\left(q_{1}^{\prime}\right)}\left(x ; q_{1}\right)}\right\},
$$

where

$$
\mathscr{L}=\log \frac{\log x}{\log Q}
$$

and $c$ is an absolute positive constant.

Proof. Choose integers $a$ and $a_{1}$ with $(a, q)=1$ and $\left(a_{1}, q_{1}\right)=1$ such that

$$
\delta(x ; q)=\max _{(b, q)=1}|\delta(x ; q, b)|=|\delta(x ; q, a)|
$$

and

$$
\delta^{\left(q_{1}^{\prime}\right)}\left(x ; q_{1}\right)=\max _{\left(b_{1}, q_{1}\right)=1}\left|\delta^{\left(q_{1}^{\prime}\right)}\left(x ; q_{1}, b_{1}\right)\right|=\left|\delta^{\left(q_{1}^{\prime}\right)}\left(x ; q_{1}, a_{1}\right)\right| .
$$


Write

$$
\begin{aligned}
& \delta_{0}=\delta(x ; q, a), \quad \delta_{0}(p)=\delta\left(\frac{x}{p} ; q, a \bar{p}\right), \\
& \delta_{1}=\delta^{\left(q_{1}^{\prime}\right)}\left(x ; q_{1}, a_{1}\right), \quad \delta_{1}(p)=\delta^{\left(q_{1}^{\prime}\right)}\left(\frac{x}{p} ; q_{1}, a_{1} \bar{p}\right), \\
& \delta_{2}=\delta^{(q)}(x ; d, a), \quad \delta_{2}(p)=\delta^{(q)}\left(\frac{x}{p} ; d, a \bar{p}\right),
\end{aligned}
$$

and set

$$
\lambda=\frac{\delta_{0}}{\delta_{1}}
$$

In this notation the estimate to be proved takes the form

$$
\delta_{0} \ll \delta_{2}+\frac{1}{\sqrt{\mathscr{L}}}(1+|\lambda|)
$$

or, equivalently,

$$
\delta_{0}^{2} \ll \delta_{0}^{2}+\frac{1}{\mathscr{L}}\left(1+|\lambda|^{2}\right)
$$

Since

$$
\sum_{Q_{1}<p \leq x^{1 / 4}} \frac{1}{p} \asymp \log \frac{\log x^{1 / 4}}{\log Q_{1}} \asymp \log \frac{\log x}{\log Q}=\mathscr{L},
$$

if $x$ is sufficiently large, as we may assume, the last relation is equivalent to

$$
\left|\delta_{0}\right|^{2} \sum \frac{1}{p} \ll\left|\delta_{2}\right|^{2} \sum \frac{1}{p}+1+|\lambda|^{2},
$$

where the sums are taken over all primes in the interval $Q_{1}<p \leq x^{1 / 4}$.

Using the inequality

$$
\left|\delta_{0}\right|^{2} \ll\left|\delta_{0}-f(p) \delta_{0}(p)\right|^{2}+|f(p)|^{2}\left|\delta_{0}(p)-\delta_{2}(p)\right|^{2}+\left|f(p) \delta_{2}(p)-\delta_{2}\right|^{2}+\left|\delta_{2}\right|^{2},
$$

we get for the left-hand side of (18) the estimate

$$
\begin{aligned}
\ll & \sum \frac{1}{p}\left|\delta_{0}-f(p) \delta_{0}(p)\right|^{2}+\sum \frac{|f(p)|^{2}}{p}\left|\delta_{0}(p)-\delta_{2}(p)\right|^{2} \\
& +\sum \frac{1}{p}\left|f(p) \delta_{2}(p)-\delta_{2}\right|^{2}+\left|\delta_{2}\right|^{2} \sum \frac{1}{p} .
\end{aligned}
$$

Of the four terms in this expression, the last is trivially bounded by the righthand side of (18). Moreover, Lemma 4 gives for the first and third terms the bound $\ll 1$, which is sufficient. It remains therefore to estimate the second term by the right-hand side of (18). We shall show that

$$
\sum \frac{1}{p}\left|\delta_{0}(p)-\delta_{2}(p)\right|^{2} \ll 1+|\lambda|^{2}
$$

which is sufficient.

An application of Lemma 5 (with $q^{\prime}=q$ ) yields

$$
\sum \frac{1}{p}\left|\delta_{0}(p)-\delta_{2}(p)\right|^{2} \ll 1+|\lambda|^{2}+\sum \frac{1}{p}\left|\delta_{0}(p)-\lambda \delta_{1}(p)\right|^{2},
$$


so that it suffices to estimate the last sum by $\ll 1+|\lambda|^{2}$. For any prime $p$ in this sum we have

$$
\begin{aligned}
\left|\delta_{0}(p)-\lambda \delta_{1}(p)\right|^{2} \ll & \left(1-|f(p)|^{2}\right)\left(\left|\delta_{0}(p)\right|^{2}+|\lambda|^{2}\left|\delta_{1}(p)\right|^{2}\right) \\
& +\left|f(p) \delta_{0}(p)-\delta_{0}\right|^{2}+\left|\delta_{0}-\lambda f(p) \delta_{1}(p)\right|^{2} .
\end{aligned}
$$

Using the bound (10) for the functions $\left|\delta_{i}(p)\right|$ and the definition of $\lambda$, this is seen to be

$$
\ll\left(1-|f(p)|^{2}\right)\left(1+|\lambda|^{2}\right) e^{-S}+\left|f(p) \delta_{0}(p)-\delta_{0}\right|^{2}+|\lambda|^{2}\left|\delta_{1}-f(p) \delta_{1}(p)\right|^{2},
$$

where $S$ is defined by (9). Thus,

$$
\begin{aligned}
& \sum \frac{1}{p}\left|\delta_{0}(p)-\lambda \delta_{1}(p)\right|^{2} \ll\left(1+|\lambda|^{2}\right) e^{-S} \sum \frac{1-|f(p)|^{2}}{p}+\sum \frac{1}{p}\left|f(p) \delta_{0}(p)-\delta_{0}\right|^{2} \\
& +|\lambda|^{2} \sum \frac{1}{p}\left|\delta_{1}-f(p) \delta_{1}(p)\right|^{2} \\
& \ll\left(1+|\lambda|^{2}\right) e^{-S} S+1+|\lambda|^{2} \ll 1+|\lambda|^{2}
\end{aligned}
$$

by two further applications of Lemma 4. The desired bound (19) now follows. Proof of the theorem. In the notation of the previous lemma we have to show that, with suitable numbers $q_{1} \geq 2$ and $q-2 \geq 2$, the bound

$$
\delta(x ; q) \ll \frac{1}{\sqrt{\mathscr{L}}}
$$

holds for all moduli $q \leq Q$ that are not divisible by $q_{1}$ or $q_{2}$.

We choose $q_{2}$ as a prime divisor of the exceptional modulus $q_{0}$ of Lemma 2 , and define $q_{1}$ as follows. Consider the inequality

$$
\max _{\substack{q^{\prime} \leq Q \\ q \mid q^{\prime}}} \delta^{\left(q^{\prime}\right)}(x ; q) \leq \frac{2 c}{\sqrt{\mathscr{L}}},
$$

where $c$ is the constant of Lemma 6. If (21) holds for all moduli $q \leq Q$ with $q_{2} \nmid q$, then taking $q_{1}=q_{2}$, we obtain the assertion of the theorem, namely the estimate (20) for all $q \leq Q$ with $q_{1} \nmid q$ and $q_{2} \nmid q$. Otherwise we choose $q_{1}$ as the smallest among those moduli $q \leq Q$ that are not divisible by $q_{2}$ and for which (21) fails. Since, by (7) and (6),

$$
\delta^{\left(q^{\prime}\right)}(x ; 1)=\frac{\varphi(q)}{\varphi\left(q^{\prime}\right)} \sum_{\left(a^{\prime}, q^{\prime}\right)=1} \delta\left(x ; q^{\prime}, a^{\prime}\right)=0,
$$

we have necessarily $q_{1} \geq 2$. We shall show that then (20) holds for any $q \leq Q$ with $q_{1} \nmid q$ and $q_{2} \nmid q$.

By the definition of $q_{1}$ there exists a positive integer $q_{1}^{\prime} \leq Q$ such that $q_{1} \mid q_{1}^{\prime}$ and $\delta^{\left(q_{1}^{\prime}\right)}\left(x ; q_{1}\right)>2 c / \sqrt{\mathscr{L}}$. Let now $q \leq Q$ be given and suppose that $q_{2} \nmid q$. 
Then we have $q_{0}+\left[q, q_{1}\right]$, since $q_{2}$ is a prime divisor of $q_{0}$, but not of $q_{1}$. Thus we can apply Lemma 6 with $q_{1}^{\prime}$ as above, and obtain, with $d=\left(q, q_{1}\right)$,

$$
\begin{aligned}
\delta(x ; q) & \leq c\left\{\delta^{(q)}(x ; d)+\frac{1}{\sqrt{\mathscr{L}}}+\frac{\delta(x ; q)}{\sqrt{\mathscr{L}} \delta^{\left(q_{1}^{\prime}\right)}\left(x ; q_{1}\right)}\right\} \\
& \leq c\left\{\delta^{(q)}(x ; d)+\frac{1}{\sqrt{\mathscr{L}}}+\frac{1}{2 c} \delta(x ; q)\right\},
\end{aligned}
$$

and hence

$$
\delta(x ; q) \leq 2 c \delta^{(q)}(x ; d)+\frac{2 c}{\sqrt{\mathscr{L}}} .
$$

If, in addition, $q$ is not divisible by $q_{1}$, then we have $d<q_{1}$, and by our choice of $q_{1}$ we conclude that (21) holds with $d$ and $q$ in place of $q_{1}$ and $q_{1}^{\prime}$, respectively. For such $q$ we therefore obtain $\delta(x ; q) \leq\left(4 c^{2}+2 c\right) / \sqrt{\mathscr{L}}$, and hence the desired estimate $(20)$. The proof of the theorem is now complete.

\section{REFERENCES}

1. E. Bombieri, Le grand crible dans la théorie analytique des nombres (seconde édition revue et augmentée), Astérisque 18 (1987/1974).

2. E. Bombieri, J. B. Friedlander and H. Iwaniec, Primes in arithmetic progressions to large moduli, Acta Math. 156 (1986), 203-251.

3. P. D. T. A. Elliott, Arithmetic functions and integer products, Springer-Verlag, New York, 1985.

4. __ Additive arithmetic functions on arithmetic progressions, Proc. London Math. Soc. 54 (1987), 15-37.

5. __ Multiplicative functions on arithmetic progressions, Mathematika 34 (1987), 199-206.

6. __ Multiplicative functions on arithmetic progressions II, Mathematika 35 (1988), 38-50.

7. P. X. Gallagher, A large sieve density estimate near $\sigma=1$, Invent. Math. 11 (1970), 329-339.

8. A. Hildebrand, Additive functions on arithmetic progressions, J. London Math. Soc. (2) 34 (1986), 394-402.

9. Y. Motohashi, An induction principle for the generalization of Bombieri's prime number theorem, Proc. Japan Acad. 52 (1976), 273-275.

10. P. Shiu, A Brun-Titchmarsh theorem for multiplicative functions, J. Reine Angew. Math. 313 (1980), 161-170.

11. E. Wirsing, Additive functions with restricted growth on the numbers of the form $p+1$, Acta Arith. 37 (1980), 345-357.

12. D. Wolke, Die mittlere Verteilung zahlentheoretischer Funktionen auf Restklassen I, J. Math. Ann. 202 (1973), 1-25.

Department of Mathematics, University of Illinois, Urbana, Illinois 61801 